\title{
Charge transport calculations by a wave-packet dynamical approach using maximally localized Wannier functions based on density functional theory: Application to high-mobility organic semiconductors
}

\author{
Hiroyuki Ishii ${ }^{1,2}$ Nobuhiko Kobayashi ${ }^{1}$ and Kenji Hirose ${ }^{3}$ \\ ${ }^{1}$ Institute of Applied Physics and Tsukuba Research Center for Interdisciplinary Materials Science, University of Tsukuba, \\ 1-1-1 Tennodai, Tsukuba, Ibaraki 305-8573, Japan \\ ${ }^{2}$ JST-PRESTO, University of Tsukuba, 1-1-1 Tennodai, Tsukuba, Ibaraki 305-8573, Japan \\ ${ }^{3}$ Smart Energy Research Laboratories, NEC Corporation, 34 Miyukigaoka, Tsukuba, Ibaraki 305-8501, Japan
}

(Received 26 January 2016; revised manuscript received 29 December 2016; published 30 January 2017)

\begin{abstract}
We present a wave-packet dynamical approach to charge transport using maximally localized Wannier functions based on density functional theory including van der Waals interactions. We apply it to the transport properties of pentacene and rubrene single crystals and show the temperature-dependent natures from bandlike to thermally activated behaviors as a function of the magnitude of external static disorder. We compare the results with those obtained by the conventional band and hopping models and experiments.
\end{abstract}

DOI: 10.1103/PhysRevB.95.035433

\section{INTRODUCTION}

Recently, understanding of the quantum transport of flexible materials, such as organic crystals, DNA, and biomaterials, has attracted considerable attention [1,2]. In contrast to covalently bonded inorganic materials, such as silicon where the crystal structure is fixed, flexible materials at room temperature can change their structures significantly, and their transport properties are determined by strong scattering with phonons due to time-dependent structural deformation. In this respect, a computational approach in which both the electronic states and the dynamical material structures are treated on an equal footing, is crucial for the understanding of intrinsic transport properties. For the charge transport calculation, we previously reported a wave-packet approach where the transport properties are evaluated using the Kubo formula on the basis of the quantum-mechanical calculations of electron wavepacket dynamics [3-5]. In this paper, we present an extended formulation of the time-dependent wave-packet diffusion (TD-WPD) method based on both density functional theory (DFT) including a recently developed formalism for van der Waals forces and the maximally localized Wannier functions (MLWFs) located on each molecule [6-10]. The intrinsic transport of flexible materials depends on the magnitudes of the intermolecular transfer energy $\gamma$ and reorganization energy $\lambda$. Ideal band transport and hopping transport are realized in the cases of $\gamma \gg \lambda$ and $\gamma \ll \lambda$, respectively. Recently reported high-mobility flexible organic semiconductors have transfer energy comparable to the reorganization energy [11] and exhibit a crossover from the hopping to bandlike transport regimes [12]. Observation of the Hall effect implies the phase coherence of carriers in organic materials [13,14]. Since $\gamma$ is strongly dependent on the electron-phonon (e-ph) interaction, it is essential to obtain accurate e-ph coupling parameters. Here we present the TD-WPD method to treat strong e-ph couplings from $a b$ initio calculations using MLWFs in detail. It is important to note that transport properties of flexible materials observed in experiments are not only explained from the intrinsic effects, but also explained from the the extrinsic effects, such as static disorder or trap potentials, and cannot be ignored [15]. The TD-WPD approach enables us to carry out transport calculations including the strong e-ph couplings and static disorder on equal footing without perturbative treatment. In previous papers, we discussed the temperature dependence of the mobility and Hall effect in competition among thermal structural fluctuations, polaron formation, and static disorder [4,5] by using the dimer approach with DFT-D formalism [16] for van der Waals interactions where the electronic state of a dimer is projected to the molecular orbitals of single molecules [17]. Here, using a more rigorous calculation method based on DFT, we obtain the intermolecular transfer energy without any fitting or projection in the most natural way.

As a demonstration, we apply the present TD-WPD method to pentacene and rubrene single crystals, organic semiconductors which are promising flexible materials [18-20] for plastic electronics fabricated by a large-area low-cost printing process $[21,22]$. We evaluate the temperature-dependent behavior of the hole mobility and the effects of the static disorder then compare the results with those obtained by the experiments and the conventional band and hopping models.

\section{CAlCulation Method}

In the TD-WPD approach, we compute the mobility of a charge $q$ along the $\xi$ direction for a material with volume $\Omega$ using the following Kubo formula:

$$
\begin{aligned}
\mu_{\xi}= & \lim _{t \rightarrow+\infty} \frac{q}{n} \int_{-\infty}^{+\infty} d E\left(-\frac{d f}{d E}\right) \\
& \times\left\langle\frac{\delta\left(E-\hat{H}_{e}\right)}{\Omega} \frac{\{\hat{\xi}(t)-\hat{\xi}(0)\}^{2}}{t}\right\rangle,
\end{aligned}
$$

where $n$ is the concentration of charge carriers $n=$ $\int d E f(E)\left\langle\delta\left(E-\hat{H}_{e}\right)\right\rangle / \Omega$. We note that the effects of strong e-ph couplings in transport are introduced in $\hat{H}_{e}$ through thermal structural fluctuations of molecules and are obtained by combining with a classical real-time molecular-dynamics simulation [23]. The Heisenberg picture of the position operator is defined by $\hat{\xi}(t)=\hat{U}^{\dagger}(t) \hat{\xi} \hat{U}(t)$, where $\hat{U}\left(t=N_{t} \Delta t\right) \equiv \Pi_{n=0}^{N_{t}-1} \exp \left\{i \hat{H}_{e}(n \Delta t) \Delta t / \hbar\right\}$ is the time-evolution operator. The quantity $\langle\cdots\rangle$ is evaluated as 
$\sum_{m=1}^{N_{w p}}\left\langle\Psi_{m}(0)|\cdots| \Psi_{m}(0)\right\rangle / N_{w p}$, where $N_{w p}$ is the number of random-phase wave-packet $\left|\Psi_{m}(0)\right\rangle$. Note that, when the Fermi distribution function is approximated as $f(E) \simeq$ $e^{-\beta\left(E-E_{F}\right)}$, we can reproduce the well-known Einstein relation $\mu_{\xi}=q D_{\xi} / k_{B} T$ from Eq. (1) where the diffusion coefficient $D_{\xi}$ is defined as

$$
D_{\xi} \equiv \lim _{t \rightarrow+\infty}(1 / t)\left[\int d E f(E)\left\langle\delta\left(E-\hat{H}_{e}\right)\{\hat{\xi}(t)-\hat{\xi}(0)\}^{2}\right\rangle\right] /
$$

$\left[\int d E f(E)\left\langle\delta\left(E-\hat{H}_{e}\right)\right\rangle\right]$.

A general Hamiltonian for electrons interacting with lattice phonons (intermolecular vibrations) is given as

$$
\hat{H}_{\text {tot }}=\sum_{n, m} \gamma_{n m} \hat{a}_{n}^{\dagger} \hat{a}_{m}+\sum_{\lambda, \mathbf{q}} \hbar \omega_{\lambda \mathbf{q}}\left(\hat{b}_{\lambda \mathbf{q}}^{\dagger} \hat{b}_{\lambda \mathbf{q}}+\frac{1}{2}\right),
$$

where $\hat{a}_{n}^{\dagger}$ and $\hat{b}_{\lambda \mathbf{q}}^{\dagger}$ represent the creation operator of electrons at the $n$th site and the creation operator of phonons with mode $\lambda$, wave-vector $\mathbf{q}$, and the vibration frequency $\omega_{\lambda \mathbf{q}}$. The transfer energies including the e-ph coupling are defined by

$$
\gamma_{n m}=\gamma_{n m}^{0}+\sum_{\lambda, \mathbf{q}} \hbar \omega_{\lambda \mathbf{q}} g_{n m}^{\lambda \mathbf{q}}\left(\hat{b}_{\lambda \mathbf{q}}^{\dagger}+\hat{b}_{\lambda-\mathbf{q}}\right)
$$

with the dimensionless e-ph coupling constant $g_{n m}^{\lambda \mathbf{q}} \equiv$ $\sum_{k, s}\left(2 \hbar M N \omega_{\lambda \mathbf{q}}^{3}\right)^{-1 / 2} e^{i \mathbf{q} \mathbf{R}_{k}}\left(\partial \gamma_{n m} / \partial \mathbf{R}_{k s}\right) \mathbf{e}_{s}^{\lambda \mathbf{q}}$, where $\mathbf{e}_{s}^{\lambda \mathbf{q}}$ is an eigenvector of the lattice phonons and $M$ and $N$, respectively, represent the mass of a single molecule and the number of unit cells $[24,25]$. Here $\gamma_{n m}^{0}$ is the transfer energy at the equilibrium position of molecules.

In the present TD-WPD method, we extract the electron (hole) Hamiltonian matrix of any flexible material by calculating MLWFs centered on a site $\mathbf{R}_{n}$ as follows:

$$
W_{\alpha \mathbf{R}_{n}}(\mathbf{r})=\frac{V}{(2 \pi)^{3}} \int_{B Z}\left\{\sum_{\beta} U_{\alpha \beta}^{(\mathbf{k})} \psi_{\beta \mathbf{k}}(\mathbf{r})\right\} e^{-i \mathbf{k} \mathbf{R}_{n}} d^{3} k,
$$

where $V$ is the volume of the unit cell and the Bloch states $\psi_{\alpha \mathbf{k}}(\mathbf{r})$ with band index $\alpha$ and wave-vector $\mathbf{k}$ are obtained by DFT including van der Waals interactions for the Kohn-Sham Hamiltonian $\hat{H}_{K S}$. The unitary matrix $\mathbf{U}^{(\mathbf{k})}$ is determined so that it minimizes the spread function $S=$ $\sum_{\alpha}\left\{\left\langle W_{\alpha \mathbf{0}}\left|r^{2}\right| W_{\alpha \mathbf{0}}\right\rangle-\left|\left\langle W_{\alpha \mathbf{0}}|\mathbf{r}| W_{\alpha \mathbf{0}}\right\rangle\right|^{2}\right\}$ [26]. Since the MLWF is located on each molecule, the transfer energies including e-ph couplings in Eq. (3) between molecules are obtained as

$$
\gamma_{n m}=\left\langle W_{\alpha \mathbf{R}_{n}}\left|\hat{H}_{K S}\right| W_{\alpha \mathbf{R}_{m}}\right\rangle .
$$

To reduce the calculation cost, we adopt the semiclassical approximation to evaluate the lattice phonons. The phonon operators in Eq. (3) are replaced by the displacements of molecules $[4,23]$ where the displacement of the $s$ th molecule in the $k$ th unit cell is defined as $\Delta \mathbf{R}_{k s}=\sum_{\lambda, \mathbf{q}} X_{\lambda \mathbf{q}} e^{i \mathbf{q} \mathbf{R}_{k}} \mathbf{e}_{s}^{\lambda \mathbf{q}}$ with $X_{\lambda \mathbf{q}}=\sqrt{\hbar / 2 M N \omega_{\lambda \mathbf{q}}}\left(\hat{b}_{\lambda \mathbf{q}}^{\dagger}+\hat{b}_{\lambda-\mathbf{q}}\right)$. Furthermore, we assume that the transfer energy $\gamma_{n m}$ depends solely on the relative coordinate $\mathbf{R}_{n m} \equiv \mathbf{R}_{m}-\mathbf{R}_{n}$, then the transfer energy in Eq. (3) is rewritten as $\gamma_{n m} \simeq \gamma_{n m}^{0}+\left(\partial \gamma_{n m} / \partial \mathbf{R}_{n m}\right) \Delta \mathbf{R}_{n m}$, where $\Delta \mathbf{R}_{n m}$ represents the change in $\mathbf{R}_{n m}$ due to intermolecular vibrations; $\left(\partial \gamma_{n m} / \partial \mathbf{R}_{n m}\right)$ is hereafter called the e-ph coupling constant. The motion of the $n$th molecule is given by the canonical equation of motion $M \Delta \ddot{\mathbf{R}}_{n}=-\partial E_{\mathrm{tot}}\left(\left\{\Delta \mathbf{R}_{i j}\right\}\right) / \partial \Delta \mathbf{R}_{n}$, where $E_{\text {tot }}$ is the total energy within a rigid molecule approximation
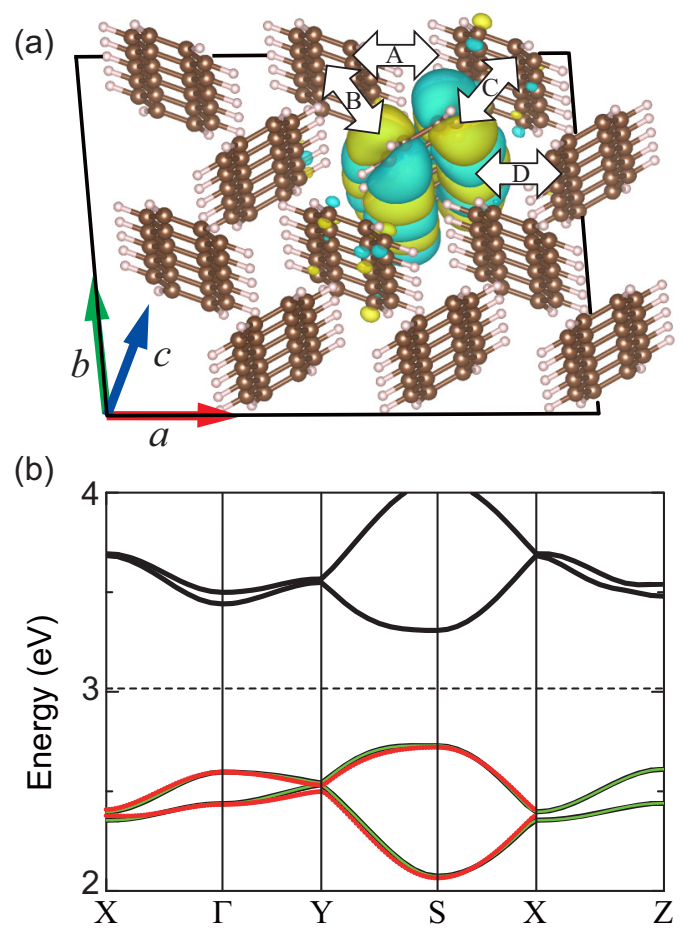

FIG. 1. (a) Structure of a single crystal of pentacene with a calculated MLWF generated from the HOMO band states in a $3 \times 2 \times 1$ supercell. The pairs of intermolecular interactions are indicated by arrows labeled A-D. (b) Energy bands obtained from DFT using the plane-wave basis set with symmetry points of $\Gamma(0,0,0), X(1 / 2,0,0), Y(0,1 / 2,0), Z(0,0,1 / 2)$, and $S(1 / 2,1 / 2,0)$. The HOMO bands obtained from MLWFs are shown by green lines. The red lines represent the bands when considering only the transfer energies labeled A-D. The broken line represents the Fermi energy.

[23]. By extracting $\Delta \mathbf{R}_{n m}$ at each time step of the moleculardynamics calculations, we can introduce the effects of thermal structural fluctuation as the ever-changing transfer energy and obtain the mobility from Eq. (1). Since the calculations of the MLWFs in the trajectory of wave packets every time step for the fluctuating lattice are quite time consuming, we employ the Taylor expansion forms for $\gamma_{n m}$.

\section{RESULTS AND DISCUSSION}

\section{A. Transport properties of pentacene and rubrene single crystals}

As a demonstration, we apply the present TD-WPD method to the transport of pentacene and rubrene single crystals [27] with high mobility. The cell parameters as well as the internal degrees of freedom are fully relaxed using the van der Waals density functional (vdW-DF) [28-33]. After obtaining the relaxed structure, we calculate MLWFs constructed from Bloch states of the highest occupied molecular orbital (HOMO) band for a $3 \times 2 \times 1$ supercell with the $\Gamma$ point in $k$ space [34]. Here we use a monolayer structure since we investigate the conduction on the $a b$ plane, which is shown in Figs. 1(a) and 2(a) with one of the calculated Wannier functions for pentacene and rubrene crystals, respectively. From Figs. 1(b) and 2(b), we can confirm that the band structure obtained from the MLWF 
(a)

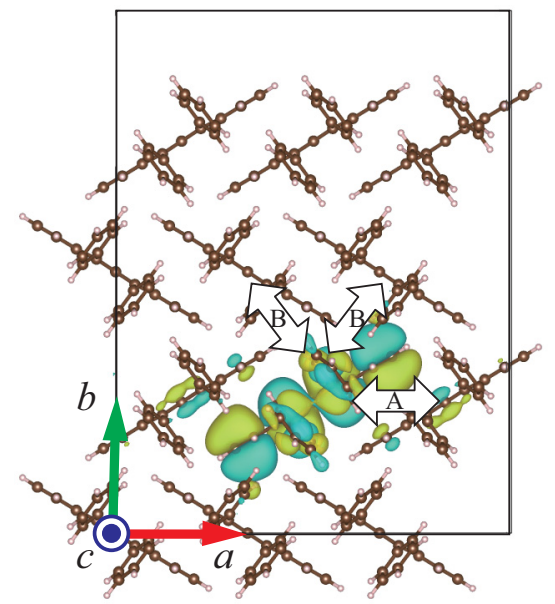

(b)

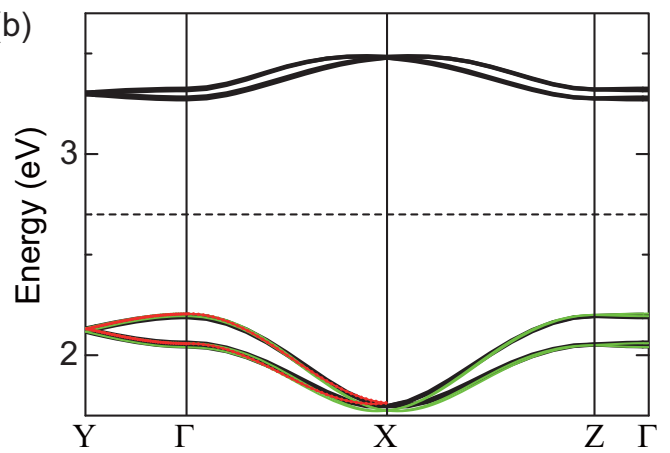

FIG. 2. (a) Structure of a single crystal of rubrene with a calculated MLWF generated from the HOMO band states in a $3 \times 2 \times 1$ supercell. The pairs of intermolecular interactions are indicated by arrows labeled A and B. (b) Energy bands obtained from DFT using the plane-wave basis set with symmetry points of $\Gamma(0,0,0), X(1 / 2,0,0), Y(0,1 / 2,0)$, and $Z(0,0,1 / 2)$. The HOMO bands obtained from MLWFs are shown by green lines. The red lines represent the bands when considering only the transfer energies labeled $\mathrm{A}$ and $\mathrm{B}$. The broken line represents the Fermi energy.

basis set (green lines) reproduces the HOMO band structure calculated using the plane-wave basis set (black lines).

Figure 3 shows the transfer energies $\gamma_{n m}$ of pentacene single crystal calculated by Eq. (5) for pairs A-D in Fig. 1(a) as a function of the displacement along the (a) in-plane direction and (b) out-of-plane directions. Similarly, the transfer energies of a rubrene single crystal for pairs A and B in Fig. 2(a) are shown in Fig. 4. Since previous experimental data obtained from translation-libration-screw analysis revealed standard deviations of the molecular displacement of about $0.2 \AA$ at room temperature [35], which means that the maximum amplitudes of intermolecular vibrations can reach up to three or four times the standard deviations, we obtain the e-ph coupling constants by fitting the change in transfer energy with a range of displacements from -0.6 to $+0.6 \AA$ using the rigid molecule approximation taking two different translational directions for $\mathbf{R}_{n m}$ into account. One is the in-plane direction along the line connecting the centers of mass. The other is the out-of-plane direction which is along the long axis of the single molecule for the pentacene crystal and along the $c$ axis for the rubrene crystal. The Taylor expansions for $\gamma_{n m}$ for the
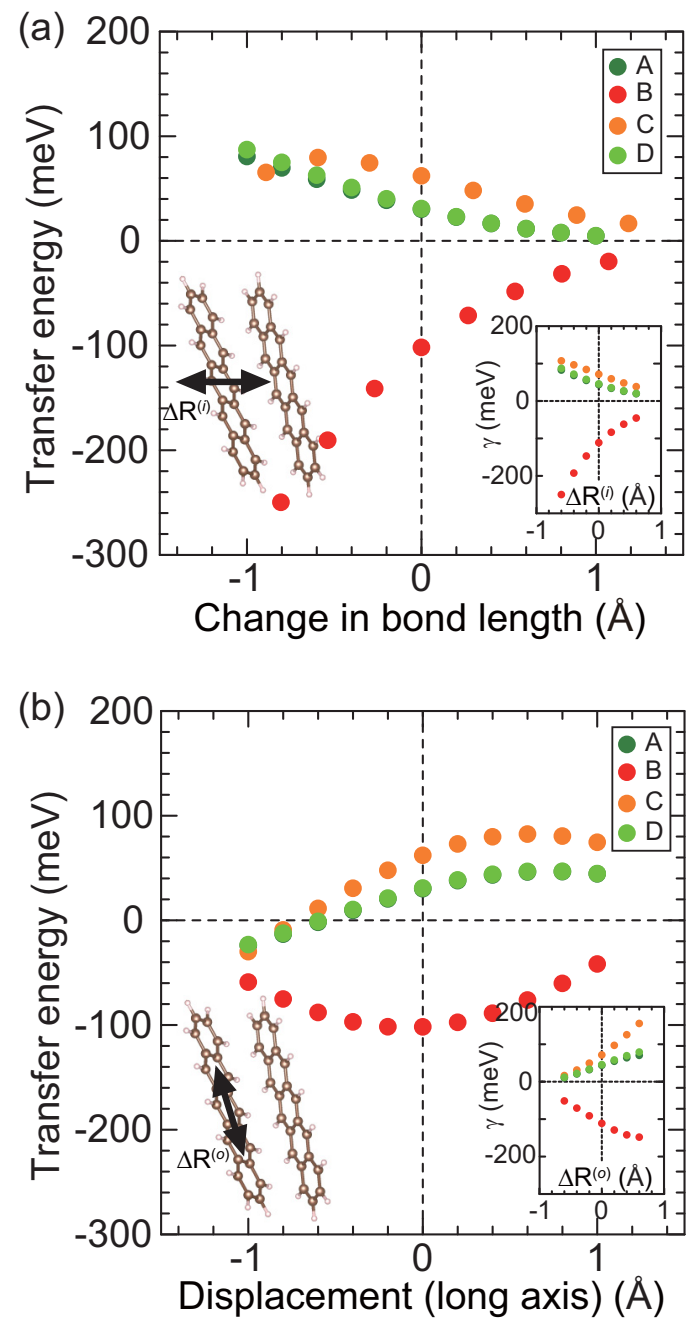

FIG. 3. Transfer energies of pentacene single crystal calculated using a MLWF basis set as a function of (a) $\Delta R^{(i)}$ and (b) $\Delta R^{(o)}$. The directions of $\Delta R^{(i)}$ and $\Delta R^{(o)}$ for pair $\mathrm{B}$ are shown by black arrows. (The inset) Transfer energies calculated by the dimer method.

pentacene crystal are found to be expressed as a second-order equation in $\Delta R_{n m}$,

$$
\begin{aligned}
\gamma_{n m} \simeq & \gamma_{n m}^{0}+\alpha_{n m}^{(i)} \Delta R_{n m}^{(i)} \\
& +\alpha_{n m}^{(o 1)} \Delta R_{n m}^{(o)}+\alpha_{n m}^{(o 2)}\left(\Delta R_{n m}^{(o)}\right)^{2},
\end{aligned}
$$

where $\Delta R_{n m}^{(i)}$ and $\Delta R_{n m}^{(o)}$ are the displacement along the inplane and out-of-plane directions. Note that the second-order term $\alpha_{n m}^{(o 2)}$ is typical for flexible organic semiconductors, not included in the perturbative approach. For comparison, the transfer energies $\gamma^{0}$ derived from the dimer method [17] and the coupling constants $\alpha^{(i)}$ in the insets of Figs. 3 and 4 show good agreement within $10 \mathrm{meV}$, whereas $\alpha^{(o 1)}$ and $\alpha^{(02)}$ of pentacene obtained by the dimer method slightly overestimate the change in transfer energies.

First, we studied the mobility of the pentacene single crystal along the $a$ axis as a function of temperature. As shown by green triangles in Fig. 5, experimental data $[15,36]$ indicate that the appearance of thermally activated transport is in many instances more likely due to the presence of extrinsic 

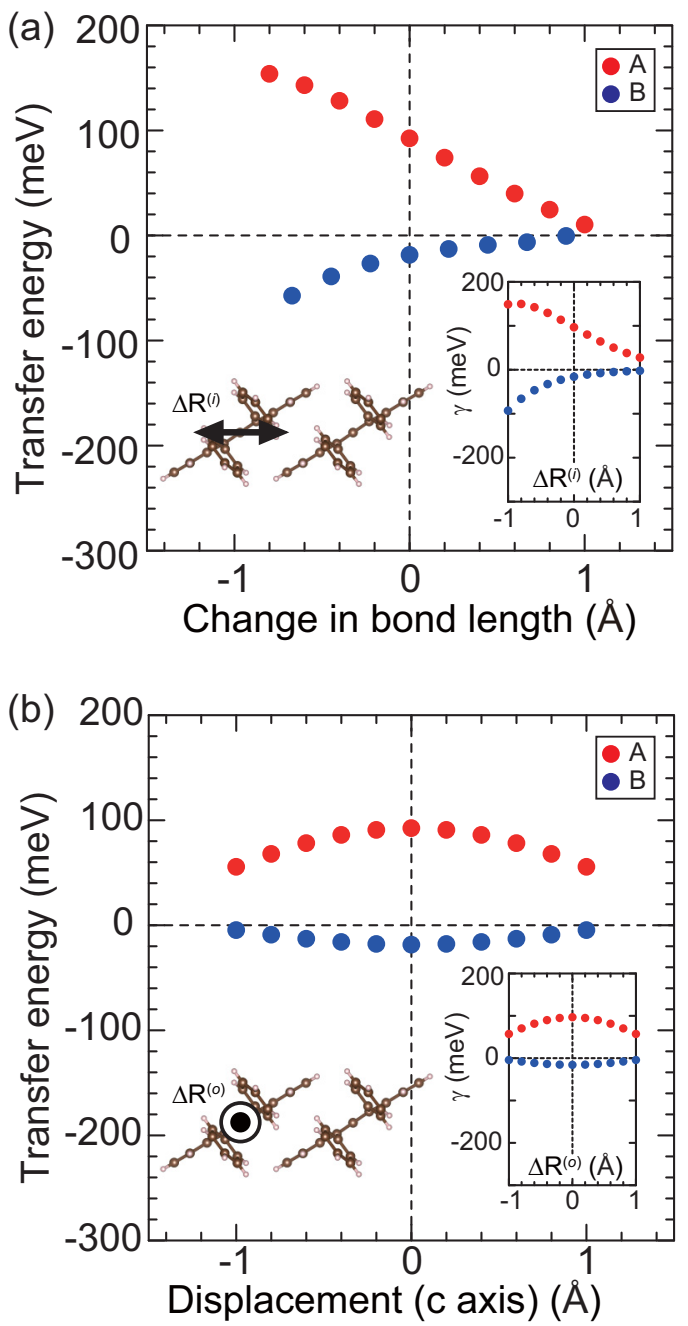

FIG. 4. Transfer energies of rubrene single crystal calculated using a MLWF basis set as a function of (a) $\Delta R^{(i)}$ and (b) $\Delta R^{(o)}$. The directions of $\Delta R^{(i)}$ and $\Delta R^{(o)}$ for pair $\mathrm{B}$ are shown by black arrows. (The inset) Transfer energies calculated by the dimer method.

static disorder with potential depths of approximately $50 \mathrm{meV}$ than to the intrinsic signature of polaron formation [37]. To compare with experiments, we introduce the extrinsic effects of on-site static disorder potentials with a Gaussian distribution having energy width $W$ from 40 to $100 \mathrm{meV}$, which are comparable to $\gamma^{0}$, showing that the motion of charge carriers is strongly disturbed by the static disorder. The intrinsic mobility for $W=0$ exhibits typical bandlike behavior, a power-law temperature dependence as shown by open circles. The $W$ dependence of the mobility shows that the intrinsic mobility of $54.4 \mathrm{~cm}^{2} \mathrm{~V}^{-1} \mathrm{~s}^{-1}$ at $300 \mathrm{~K}$ is decreased to $14.9 \mathrm{~cm}^{2} \mathrm{~V}^{-1} \mathrm{~s}^{-1}$ for $W=40 \mathrm{meV}$ and to $1.1 \mathrm{~cm}^{2} \mathrm{~V}^{-1} \mathrm{~s}^{-1}$ for $W=100 \mathrm{meV}$ by the existence of static disorder. The variation in the mobilities at room temperature reported in experimental works of pentacene-single-crystal transistors are shown by the green bar [38-42]. Furthermore, we obtained a change in the temperature dependence from power-law dependence to thermally activated behavior via temperatureindependent behavior with $5-10 \mathrm{~cm}^{2} \mathrm{~V}^{-1} \mathrm{~s}^{-1}$ at approximately $W=50 \mathrm{meV}$. In previous experimental reports,

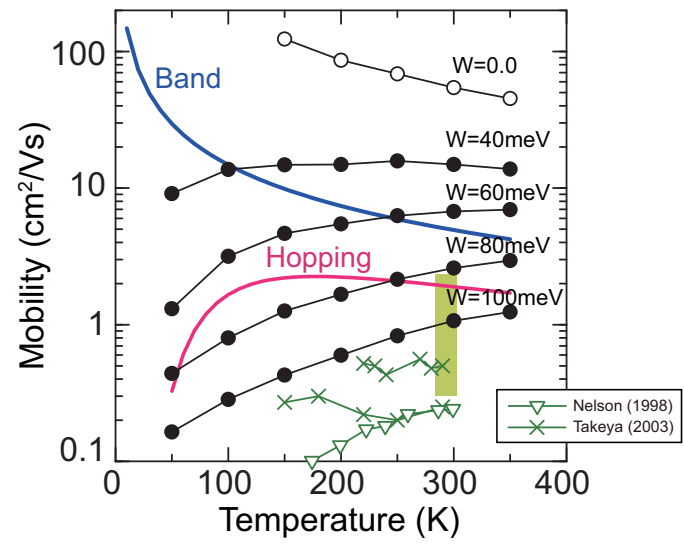

FIG. 5. Calculated mobility of pentacene single crystal along the $a$ axis as a function of temperature for several magnitudes of static disorder $W$ from 0 to $100 \mathrm{meV}$. The band mobility and hopping mobility also are shown by blue and red lines, respectively. For comparison, the green bar represents the variation in the mobilities at room temperature reported in several experimental works of pentacene-single-crystal transistors [38-42]. Experimentally observed temperature-dependent mobilities of thin-film transistors [36] and single-crystal transistors [43] are plotted by green triangles and crosses, respectively.

temperature-independent mobility of $0.5-1 \mathrm{~cm}^{2} \mathrm{~V}^{-1} \mathrm{~s}^{-1}$ is often observed in pentacene-single-crystal devices as shown by green crosses in Fig. 5 [43].

Next we investigated the transport properties of rubrene single crystal with the highest mobility in organic semiconductors. Figure 6 shows the mobility as a function of temperature for several magnitudes of static disorder $W$ from 0 to $100 \mathrm{meV}$. The intrinsic mobility of $192 \mathrm{~cm}^{2} \mathrm{~V}^{-1} \mathrm{~s}^{-1}$ at $300 \mathrm{~K}$ is decreased to $75.3 \mathrm{~cm}^{2} \mathrm{~V}^{-1} \mathrm{~s}^{-1}$ for $W=40 \mathrm{meV}$ and to $3.62 \mathrm{~cm}^{2} \mathrm{~V}^{-1} \mathrm{~s}^{-1}$ for $W=100 \mathrm{meV}$. Experimentally observed mobilities at room temperature of rubrene single-

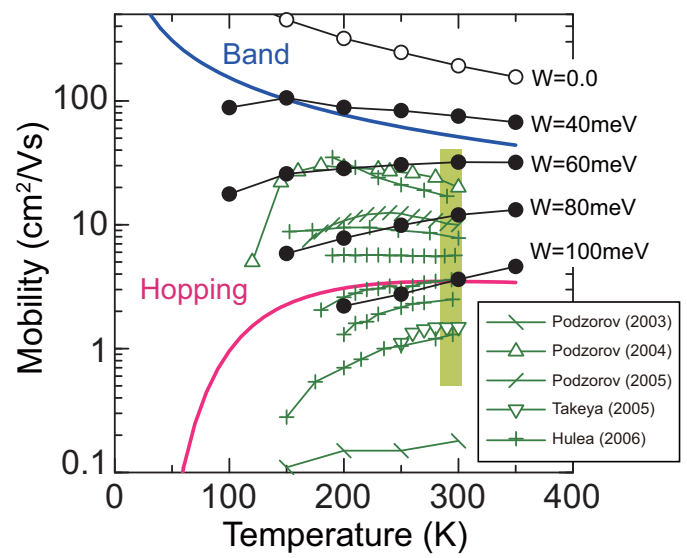

FIG. 6. Calculated mobility of a rubrene single crystal along the $a$ axis as a function of temperature for several magnitudes of static disorder $W$ from 0 to $100 \mathrm{meV}$. For comparison, temperature-dependent mobilities of rubrene-single-crystal transistors in experimental works [12-14,52,53] are plotted by green symbols. The green bar represents the variation in the mobilities at room temperature reported in previous experimental works $[39,41,44-51]$. 
crystal transistors are distributed from 0.5 to $40 \mathrm{~cm}^{2} \mathrm{~V}^{-1} \mathrm{~s}^{-1}$ as shown by the green bar $[39,41,44-51]$. The temperaturedependent mobilities [12-14,52,53] also are plotted by green symbols. These experimental results show a change in the temperature dependence from power-law to thermally activated behavior via temperature-independent behavior with 5-10 $\mathrm{cm}^{2} \mathrm{~V}^{-1} \mathrm{~s}^{-1}$. On the other hand, we can see a similar change in the temperature dependence of calculated mobilities from power-law dependence to thermally activated behavior. The temperature-independent mobility with $30 \mathrm{~cm}^{2} \mathrm{~V}^{-1} \mathrm{~s}^{-1}$ is obtained at $W=60 \mathrm{meV}$.

From the quantitative comparison between our calculated mobilities and the experimentally observed mobility, it seems that the present method overestimates the magnitude of mobilities for pentacene and rubrene single crystals. More accurate calculations are expected to be performed by considering various phonon modes, such as librational vibrations, intramolecular vibrations, and polaron effects [4,25,54-57].

\section{B. Comparison with band and hopping models}

Finally, we compare the calculated results with those obtained from two limiting conventional models, which are classified depending on the magnitudes of the intermolecular transfer energy $\gamma$ and reorganization energy $\lambda$. One is the band transport model, which assumes that electrons propagate as coherent delocalized waves scattered by the lattice phonons through crystals, which is applicable for $\gamma \gg \lambda$. The scattering rate, obtained by the acoustic deformation potential model [58], is inversely proportional to the temperature $\tau^{\text {band }}=$ $\hbar^{3} B L_{\mathrm{eff}} / \varepsilon_{a c}^{2} m_{d} k_{B} T$, where $B$ is the elastic modulus, $L_{\mathrm{eff}}$ is the effective width of carrier confinement, $\varepsilon_{a c}$ is the acoustic deformation potential, and $m_{d}$ is the density of states mass. The diffusion coefficient along the $a$ axis is obtained as $D_{a}^{\text {band }}=\int_{-\infty}^{+\infty} d t\left\langle\hat{v}_{a}(t) \hat{v}_{a}(0)\right\rangle=v_{a}^{2} \tau^{\text {band }}$, where $v_{a}$ is the group velocity. As a result, we can obtain the power-law temperature dependence of the band mobility as $\mu_{a}^{\text {band }}=q D_{a}^{\text {band }} / k_{B} T=$ $q \tau^{\text {band }} / m_{a}^{*}$, where $m_{a}^{*}$ is the effective mass along the $a$ axis of the HOMO band. Using the parameters obtained from DFT calculations [11,59], we obtain the band mobility of pentacene and rubrene single crystals shown by the blue line in Figs. 5 and 6 , respectively. Ideal coherent band transport is not realized in organic semiconductors because the HOMO band-edge states are spatially localized owing to strong e-ph couplings [55,60-62]. Although the TD-WPD method can introduce the strong e-ph interactions without the perturbative approach, the intrinsic mobility ( $W=0$ ) obtained by the TD-WPD method becomes larger than the ideal band mobility. The possible origin of the difference is suggested in some papers $[62,63]$ but still under consideration.
The other limiting conventional model is the incoherent hopping model based on the semiclassical Marcus theory $[64,65]$, which assumes that a carrier creates a small polaron state localized on a single molecule with distortion then hops to neighbor molecules without phase coherence; this model is generally applicable for $\gamma \ll \lambda$. The hopping rate from the $n$th site to the $m$ th site exhibits thermally activated behavior and is given by $1 / \tau_{n m}^{\text {hop }}=\left(\gamma_{n m}^{2} / \hbar\right) \sqrt{\pi / \lambda k_{B} T} \exp \left(-\lambda / 4 k_{B} T\right)$. The diffusion coefficient is obtained from the hopping rate $D^{\text {hop }}=$ $\left(1 / 2 n_{d}\right) \sum_{k} a_{n k}^{2} P_{n k} / \tau_{n k}^{\text {hop }}$, where $P_{n m}=\left(1 / \tau_{n m}^{\text {hop }}\right) / \sum_{k}\left(1 / \tau_{n k}^{\text {hop }}\right)$, $a$ is the intermolecular distance, and $n_{d}$ is the dimensionality. As a result, we can obtain the hopping mobility with thermally activated behavior $\mu^{\text {hop }}=q D^{\text {hop }} / k_{B} T$. Interestingly, the calculated hopping mobility shown by the red lines in Figs. 5 and 6 exhibits temperature-independent mobility around room temperature with a mobility of $\sim 1 \mathrm{~cm}^{2} \mathrm{~V}^{-1} \mathrm{~s}^{-1}$. However, we should note that the parameter region where this perturbative approach is applicable is $\lambda / 4 \gg \gamma$, whereas $\gamma$ is comparable to $\lambda$ for pentacene and rubrene single crystals [11]. This supports the idea that the temperature-independent mobility is induced by the presence of extrinsic disorder [66,67].

\section{SUMMARY}

To summarize, we present a methodology of the wavepacket dynamical approach to the charge transport of flexible materials using maximally localized Wannier functions based on the density functional theory including accurate van der Waal interactions. The strong electron-phonon interactions due to thermal fluctuation are appropriately included as intrinsic effects. As a demonstration, we study the transport properties of pentacene and rubrene organic semiconductors. We compare the calculated temperature-dependent behavior of mobilities with experimental results. We also find the temperature-dependent behavior from bandlike to thermally activated behavior due to extrinsic disorder effects, which correspond to experimental observations.

\section{ACKNOWLEDGMENTS}

We thank S. Yanagisawa, I. Hamada, and J. Takeya for valuable comments and suggestions. This work was supported by the JST-PRESTO program "Molecular technology and creation of new functions." We also acknowledge JSPS KAKENHI Grants No. 15H05418 and No. 26105011. Numerical calculations were performed at the Supercomputer Center, ISSP, University of Tokyo.
[1] A. Troisi, D. L. Cheung, and D. Andrienko, Phys. Rev. Lett. 102, 116602 (2009).

[2] R. Gutiérrez, R. A. Caetano, B. P. Woiczikowski, T. Kubar, M. Elstner, and G. Cuniberti, Phys. Rev. Lett. 102, 208102 (2009).

[3] H. Ishii, N. Kobayashi, and K. Hirose, Appl. Phys. Express 1, 123002 (2008); Phys. Rev. B 82, 085435 (2010).
[4] H. Ishii, K. Honma, N. Kobayashi, and K. Hirose, Phys. Rev. B 85, 245206 (2012).

[5] H. Ishii, H. Tamura, M. Tsukada, N. Kobayashi, and K. Hirose, Phys. Rev. B 90, 155458 (2014).

[6] We note that various approaches have been developed for the electron-phonon interaction based on DFT [7-10]. 
[7] Y. S. Lee, M. B. Nardelli, and N. Marzari, Phys. Rev. Lett. 95, 076804 (2005).

[8] F. Giustino, J. R. Yates, I. Souza, M. L. Cohen, and S. G. Louie, Phys. Rev. Lett. 98, 047005 (2007).

[9] N. Vukmirovic, C. Bruder, and V. M. Stojanovic, Phys. Rev. Lett. 109, 126407 (2012).

[10] S. Kim and N. Marzari, Phys. Rev. B 87, 245407 (2013).

[11] H. Kobayashi, N. Kobayashi, S. Hosoi, N. Koshitani, D. Murakami, R. Shirasawa, Y. Kudo, D. Hobara, Y. Tokita, and M. Itabashi, J. Chem. Phys. 139, 014707 (2013).

[12] I. N. Hulea, S. Fratini, H. Xie, C. L. Mulder, N. N. Iossad, G. Rastelli, S. Ciuchi, and A. F. Morpurgo, Nat. Mater. 5, 982 (2006).

[13] J. Takeya, K. Tsukagoshi, Y. Aoyagi, T. Takenobu, and Y. Iwasa, Jpn. J. Appl. Phys., Part 2 44, L1393 (2005).

[14] V. Podzorov, E. Menard, J. A. Rogers, and M. E. Gershenson, Phys. Rev. Lett. 95, 226601 (2005).

[15] W. L. Kalb and B. Batlogg, Phys. Rev. B 81, 035327 (2010).

[16] S. Grimme, J. Comput. Chem. 25, 1463 (2004).

[17] E. F. Valeev, V. Coropceanu, D. A. da Silva Filho, S. Salman, and J.-L. Brédas, J. Am. Chem. Soc. 128, 9882 (2006).

[18] K. Takimiya, H. Ebata, K. Sakamoto, T. Izawa, T. Otsubo, and Y. Kunugi, J. Am. Chem. Soc. 128, 12604 (2006).

[19] T. Yamamoto and K. Takimiya, J. Am. Chem. Soc. 129, 2224 (2007).

[20] T. Okamoto, C. Mitsui, M. Yamagishi, K. Nakahara, J. Soeda, Y. Hirose, K. Miwa, H. Sato, A. Yamano, T. Matsushita, T. Uemura, and J. Takeya, Adv. Mater. 25, 6392 (2013).

[21] S. R. Forrest, Nature (London) 428, 911 (2004).

[22] H. Minemawari, T. Yamada, H. Matsui, J. Tsutsumi, S. Haas, R. Chiba, R. Kumai, and T. Hasegawa, Nature (London) 475, 364 (2011).

[23] H. Ishii, N. Kobayashi, and K. Hirose, Phys. Rev. B 88, 205208 (2013).

[24] K. Hannewald and P. A. Bobbert, Phys. Rev. B 69, 075212 (2004).

[25] F. Ortmann, F. Bechstedt, and K. Hannewald, Phys. Rev. B 79, 235206 (2009).

[26] N. Marzari and D. Vanderbilt, Phys. Rev. B 56, 12847 (1997).

[27] We employ pentacene and rubrene single crystals of $360 \times$ $300 \times 1$ unit cells. The masses of pentacene and rubrene single molecules are 278.3 and $532.7 \mathrm{u}$, respectively, in the unified atomic mass unit. The number density of charge carriers in the crystal is fixed at $10^{12} \mathrm{~cm}^{-2}$. The dynamics calculations are performed using 48 initial conditions for up to 2 ps with a time step of $\Delta t \simeq 1$ fs for the evaluation of Eq. (1).

[28] P. Giannozzi, S. Baroni, N. Bonini, M. Calandra, R. Car, C. Cavazzoni, D. Ceresoli, G. L. Chiarotti, M. Cococcioni, I. Dabo, A. D. Corso, S. de Gironcoli, S. Fabris, G. Fratesi, R. Gebauer, U. Gerstmann, C. Gougoussis, A. Kokalj, M. Lazzeri, L. Martin-Samos, N. Marzari, F. Mauri, R. Mazzarello, S. Paolini, A. Pasquarello, L. Paulatto, C. Sbraccia, S. Scandolo, G. Sclauzero, A. P. Seitsonen, A. Smogunov, P. Umari, and R. M. Wentzcovitch, J. Phys.: Condens. Matter 21, 395502 (2009).

[29] For the vdW-DF [30] family, vdW-DF2-b86r [31] is used here. The cutoff energies for the plane wave and charge density are 80 and $800 \mathrm{Ry}$, and the Brillouin zone integration is performed with a $4 \times 4 \times 2 k$-point set. The obtained cell parameters are $a=6.281 \AA, b=7.574 \AA, c=14.314 \AA$, $\alpha=77.348^{\circ}, \beta=88.432^{\circ}$, and $\gamma=84.258^{\circ}$, corresponding to the low-temperature phase of a pentacene single crystal $[32,33]$.

[30] M. Dion, H. Rydberg, E. Schröder, D. C. Langreth, and B. I. Lundqvist, Phys. Rev. Lett. 92, 246401 (2004).

[31] I. Hamada, Phys. Rev. B 89, 121103(R) (2014).

[32] T. Siegrist, C. Kloc, J. H. Schön, B. Batlogg, R. C. Haddon, S. Berg, and C. A. Thomas, Angew. Chem. Int. Ed. 40, 1732 (2001).

[33] S. Yanagisawa, K. Okuma, T. Inaoka, and I. Hamada, J. Electron Spectrosc. Relat. Phenom. 204, 159 (2015).

[34] A. A. Mostofi, J. R. Yates, Y.-S. Lee, I. Souza, D. Vanderbilt, and N. Marzari, Comput. Phys. Commun. 178, 685 (2008).

[35] T. Fukami, H. Ishii, N. Kobayashi, T. Uemura, K. Sakai, Y. Okada, J. Takeya, and K. Hirose, Appl. Phys. Lett. 106, 143302 (2015).

[36] S. F. Nelson, Y.-Y. Lin, D. J. Gundlach, and T. N. Jackson, Appl Phys. Lett. 72, 1854 (1998).

[37] Y. Li, V. Coropceanu, and J.-L. Brédas, J. Phys. Chem. Lett. 3, 3325 (2012).

[38] V. Y. Butko, X. Chi, D. V. Lang, and A. P. Ramirez, Appl. Phys. Lett. 83, 4773 (2003).

[39] C. Goldmann, S. Haas, C. Krellner, K. P. Pernstich, D. J. Gundlach, and B. Batlogg, J. Appl. Phys. 96, 2080 (2004).

[40] J. Y. Lee, S. Roth, and Y. W. Park, Appl. Phys. Lett. 88, 252106 (2006).

[41] C. Reese, W.-J. Chung, M. Ling, M. Roberts, and Z. Bao, Appl. Phys. Lett. 89, 202108 (2006).

[42] T. Uemura, M. Yamagishi, J. Soeda, Y. Takatsuki, Y. Okada, Y. Nakazawa, and J. Takeya, Phys. Rev. B 85, 035313 (2012).

[43] J. Takeya, C. Goldmann, S. Haas, K. P. Pernstich, B. Ketterer, and B. Batlogg, J. Appl. Phys. 94, 5800 (2003).

[44] E. Menard, V. Podzorov, S.-H. Hur, A. Gaur, M. E. Gershenson, and J. A. Rogers, Adv. Mater. 16, 2097 (2004).

[45] A. F. Stassen, R. W. I. de Boer, N. N. Iosad, and A. F. Morpurgo, Appl. Phys. Lett. 85, 3899 (2004).

[46] V. C. Sundar, J. Zaumseil, V. Podzorov, E. Menard, R. L. Willett, T. Someya, M. E. Gershenson, and J. A. Rogers, Science 303, 1644 (2004).

[47] J. Takeya, J. Kato, K. Hara, M. Yamagishi, R. Hirahara, K. Yamada, Y. Nakazawa, S. Ikehata, K. Tsukagoshi, Y. Aoyagi, T. Takenobu, and Y. Iwasa, Phys. Rev. Lett. 98, 196804 (2007).

[48] J. Takeya, M. Yamagishi, Y. Tominari, R. Hirahara, Y. Nakazawa, T. Nishikawa, T. Kawase, T. Shimoda, and S. Ogawa, Appl. Phys. Lett. 90, 102120 (2007).

[49] C. Reese and Z. Bao, Adv. Mater. 19, 4535 (2007).

[50] K. Marumoto, N. Arai, H. Goto, M. Kijima, K. Murakami, Y. Tominari, J. Takeya, Y. Shimoi, H. Tanaka, S.-i. Kuroda, T. Kaji, T. Nishikawa, T. Takenobu, and Y. Iwasa, Phys. Rev. B 83, 075302 (2011).

[51] B. Lee, Y. Chen, D. Fu, H. T. Yi, K. Czelen, H. Najafov, and V. Podzorov, Nat. Mater. 12, 1125 (2013).

[52] V. Podzorov, V. M. Pudalov, and M. E. Gershenson, Appl. Phys. Lett. 82, 1739 (2003).

[53] V. Podzorov, E. Menard, A. Borissov, V. Kiryukhin, J. A. Rogers, and M. E. Gershenson, Phys. Rev. Lett. 93, 086602 (2004).

[54] A. Troisi, Adv. Mater. 19, 2000 (2007). 
[55] J.-D. Picon, M. N. Bussac, and L. Zuppiroli, Phys. Rev. B 75, 235106 (2007).

[56] R. C. Hatch, D. L. Huber, and H. Höchst, Phys. Rev. Lett. 104, 047601 (2010).

[57] L. Wang and D. Beljonne, J. Phys. Chem. Lett. 4, 1888 (2013).

[58] J. E. Northrup, Appl. Phys. Lett. 99, 062111 (2011).

[59] The effective mass of a pentacene single crystal reported in Ref. [11] is incorrect because the signs of the transfer integrals are not taken into account. In the present paper, we showed the band mobility of a pentacene single crystal using the effective masses of $m_{a} / m_{0}=7.00$ and $m_{b} / m_{0}=1.54$, which are evaluated from the band dispersion of Fig. 1(b). We employed the other parameters reported in Ref. [11].

[60] A. Troisi and G. Orlandi, Phys. Rev. Lett. 96, 086601 (2006).

[61] S. Fratini and S. Ciuchi, Phys. Rev. Lett. 103, 266601 (2009).

[62] S. Ciuchi, S. Fratini, and D. Mayou, Phys. Rev. B 83, 081202(R) (2011).
[63] P. V. Parandekar and J. C. Tully, J. Chem. Phys. 122, 094102 (2005).

[64] R. A. Marcus, J. Chem. Phys. 24, 966 (1956).

[65] N. S. Hush, J. Chem. Phys. 28, 962 (1958).

[66] Note that some models derived from a rigorous Hamiltonian have been proposed for the carrier transport of high-mobility organic single crystals with strong e-ph scattering. A coherent polaron model $[24,25,67]$ assumes that a charge carrier dressed by phonon clouds creates the quasiparticle band state and that coherent polarons are scattered by weak static disorder. However, transient localized states induced by thermal structural fluctuations are missing and are found to play an important role at room temperature [55,60-62]. A flexible surface-hopping model treats e-ph coupling in a nonperturbative manner based on a dephasing strategy [57]. However, it is difficult to use this model to investigate the phase coherence of carriers observed in experiments on the Hall effects [13,14].

[67] C. Motta and S. Sanvito, J. Chem. Theory Comput. 10, 4624 (2014). 